\title{
MODELO DE GESTIÓN DE CALIDAD EN PROYECTOS DE INVESTIGACIÓN Y DESARROLLO EN EL ÁMBITO DE LA UNIVERSIDAD TECNOLÓGICA NACIONAL ACORDE A LA NORMA INTERNACIONAL ISO 10006: 2003
}

\author{
LuCAS GABRiEl GimÉNEZ* \\ Recebido em: setembro de 2007 \\ Aprovado: 20/01/2008
}

*Universidad Tecnológica Nacional, Facultad Regional Avellaneda, Secretaría de Ciencia y Tecnología. Av. Ramón Franco y San Vicente, (1874), Avellaneda, Buenos Aires, Republica Argentina.

E-mail: lgimenez@fra.utn.edu.ar

Resumen: Se estudió el estado de situación de la investigación en la Argentina y, específicamente, en la Universidad Tecnológica Nacional. Se relevó la normativa que hace referencia a la investigación desde la creación de la Universidad Tecnológica Nacional hasta nuestros días. Se analizaron los comentarios formulados por la Comisión Nacional de Evaluación y Acreditación Universitaria respecto al estado de la investigación y el desarrollo en la acreditación de las carreras. A partir de una encuesta a investigadores de las Facultades Regionales Avellaneda y Buenos Aires surgió la necesidad de mejorar los formularios para la presentación de proyectos. Se analizaron distintas normativas internacionales y se concluyó que la más adecuada, para tomar como referencia, era la norma ISO 10006:2003 (Sistemas de Gestión de Calidad. Directrices para la Gestión de Calidad en Proyectos). Se estudió esta norma y se explicó la forma de interpretación de cada capítulo en el ámbito de la Universidad Tecnológica Nacional. Se realizó una adecuación de los formularios actuales y se obtuvo un modelo preliminar que fue validado en cuatro grupos de investigación. El resultado de la validación arrojó posibilidades de mejoras que se aplicaron al modelo definitivo para la presentación de proyectos.

Palabras clave: Proyectos de Investigación y Desarrollo. Norma ISO 10006:2003.

\begin{abstract}
QUALITY MANAGEMENT MODEL IN RESEARCH AND DEVELOPMENT PROJECTS IN THE AMBITO F THE NACIONAL TECHNOLOGICAL UNIVERSITY ACCORDING TO THE INTERNACIONAL REGULATION ISO 10006:2003
\end{abstract}

\begin{abstract}
This paper examines the situation of research in Argentina, specifically, at the National Technological University. The normative referred to the research since the creation of the National Technological University up to the present time. The comments formulated by the National University Evaluation and Accreditation Committee about the state of research and development in the accreditation of programs were analyzed. Based on a survey taken by researchers from the Avellaneda and Buenos Aires Regional Colleges, the need of improving the forms for presenting projects arose. Different international guidelines were analyzed and it was concluded that the most appropriate standard to be used as reference was the ISO 10006:2003 (Quality Management Systems. Guidelines for Project Quality Management). This standard was studied and the way in which to interpret each chapter at the ambit of the National Technological University was explained. The present forms were adapted and a preliminary model was obtained, which was validated in four research groups. The result of the validation showed possibilities of improvements that were applied to the final model for project presentation.
\end{abstract}

Key words: Research and Development Projects - ISO 10006:2003.

1 Trabajo realizado sobre la base de la tesis presentada por el Ing. L. G. Giménez para optar al grado de Magíster en Ingeniería en Calidad, bajo la dirección de M. M. Mazzini y R. Brunetti, con calificación sobresaliente. 


\section{Introducción}

En un mundo globalizado, las actividades de investigación y el desarrollo de proyectos de una universidad no escapan a la necesidad de comparación con las de otros sectores. Para que dicha comparación sea viable, es necesario que tengan bases comunes, lo que lleva a la necesidad de la utilización de normas internacionales.

Hoy en día es por todo el mundo ampliamente conocida la necesidad de trabajar con un sistema de gestión de la calidad para garantizar, al menos, que se han seguido procedimientos preestablecidos y acordados entre las partes.

Dentro de las distintas Facultades Regionales (FR), Centros y Unidades Académicas (UA) de la Universidad Tecnológica Nacional (UTN) se encuentran grupos de investigación y desarrollo trabajando en más de 100 proyectos, según informa la Secretaría de Ciencia y Tecnología de dicha Universidad.

Los proyectos deben ser presentados en formularios que la Secretaría de Ciencia y Tecnología (SCyT) pone a disposición en su página web (www.utn. edu.ar/scyt/proyI\&D) y que apuntan a su normalización. Sin embargo, estos formularios no forman parte de un Sistema de Gestión de la Calidad (SGC) de dicha casa de estudios. Claro está que la aplicación de un Modelo de Gestión de Calidad (MGC) a los proyectos de investigación y desarrollo no garantiza el éxito del proyecto, pero brinda la posibilidad de detectar tempranamente oportunidades de mejora y tener un adecuado control de gestión.

Para ello, lo primero que se debe definir es la normativa a aplicar en su formulación. Con la investigación realizada, se ha llegado a la conclusión de que la normativa que más se ajusta a la necesidad planteada es la Norma Internacional ISO 10006:2003, que proporciona orientación sobre la gestión de la calidad en los proyectos.

Esta norma perfila los principios y las prácticas del sistema de gestión de la calidad, cuya implementación es importante para el logro de los objetivos de la calidad en los proyectos y complementa la orientación que ofrece la Norma ISO 9004:2000 (Sistemas de Gestión de Calidad. Directrices para la mejora de desempeño).

La Norma ISO 10006:2003 está dirigida al más amplio espectro de proyectos, ya que es aplicable a cualquier formato, independientemente de su extensión y grado de complejidad. Puede ser desde un proyecto individual a uno que forme parte de un programa o cartera de proyectos.

Atento a lo anteriormente mencionado es que el objetivo del trabajo fue la confección de un Modelo de Gestión de la Calidad para los Proyectos de 
Investigación y Desarrollo, brindando así una herramienta a quienes quieran encarar sus trabajos con una concepción apuntalada en una normativa internacional de calidad.

Dado que sería imposible poder validar un modelo que cubriera todas las etapas de los proyectos, desde su presentación hasta su finalización, se decidió que el alcance del trabajo abarcaría sólo a las etapas de presentación y aprobación.

Para evaluar su aplicabilidad, el modelo preliminar fue validado y probado en proyectos de las Facultades Regionales de Avellaneda y Buenos Aires de la Universidad Tecnológica Nacional. De esas aplicaciones surgieron las mejoras a realizar que fueron aplicadas, generando así el Modelo definitivo.

El resultado de este trabajo podría ser aplicado a cualquier proyecto de Investigación y Desarrollo dentro de la UTN y de ser necesario, y con los ajustes pertinentes, a los de cualquier otro organismo o empresa.

\section{Desarrollo del Trabajo}

\section{Antecedentes de los grupos de investigación en la Universidad Tecnológica Nacional}

Si bien la UTN nace en el año 1948, es recién en 1989 cuando surge la primera Ordenanza donde se explica la necesidad de establecer el Régimen del Investigador y normar la creación y el funcionamiento de sus Centros de Investigación y Desarrollo, conforme al artículo 12 del Estatuto Universitario (Ministerio de Cultura y Educación, Universidad Tecnológica Nacional, 1998).

La ordenanza en cuestión es la número 665 (MINISTERIO DE CULTURA Y EDUCACIÓN, UNIVERSIDAD TECNOLÓGICA NACIONAL, 1989), que expresa que los Centros de Investigación y Desarrollo tienen como misión elaborar y ejecutar planes y programas de Investigación y Desarrollo en el área de su especialización.

Dos años más tarde, en la Ordenanza 703 (MINISTERIO DE CULTURA Y EDUCACIÓN, UNIVERSIDAD TECNOLÓGICA NACIONAL, 1991) se establece que será la Secretaría de Ciencia y Tecnología la responsable de propiciar la integración de la política científico-tecnológica-educativa dentro de la Universidad. Entre sus misiones y funciones figura intervenir en la formulación de las políticas en materia de investigaciones e impulsar la investigación con la finalidad de contribuir al crecimiento del país. 
En esta Ordenanza se establece que los proyectos deberán tener definidos:

a) Jefe de Grupo

b) Objetivos

c) Nomina de Integrantes

d) Plan de Trabajo

e) Metodología a utilizar

f) Tipo de Transferencia esperada

g) Equipamiento necesario, infraestructura requerida y recursos solicitados.

Con la Resolución 275 (ARGENTINA MINISTERIO DE CULTURA Y EDUCACIÓN, UNIVERSIDAD TECNOLÓGICA NACIONAL, 1995) se establece una política de investigación y desarrollo donde, entre otras cosas, se plantea como uno de los objetivos: consolidar y ampliar la actividad de investigación y desarrollo en la UTN.

Otro de los puntos que se considera importante mencionar en relación con la citada resolución, es el referente a la necesidad de alentar la participación de los grupos de investigación y desarrollo de la UTN en actividades transnacionales, particularmente con grupos homólogos del MERCOSUR y América, incluyendo el intercambio de especialistas e información.

En la Resolución 232/98 (Ministerio de Cultura y Educación, Universidad Tecnológica Nacional, 1998) se establecen los nuevos criterios de la política de ciencia y tecnología en la UTN. En este caso se intenta que el rumbo a seguir por los investigadores y la comunidad universitaria sea tal que permita armonizar y comenzar a integrarse con el Sistema Científico Tecnológico Nacional. Concretamente, en una de sus partes establece

La UTN atenderá prioritariamente a la investigación aplicada y al Desarrollo Tecnológico e Innovación que le sea requerida por el Estado Nacional, las Provincias y Municipios y el sector productor de bienes y servicios permitiendo satisfacer el concepto insito a la ingeniería de utilizar en forma económica los materiales y fuerzas de la naturaleza en beneficio de la humanidad.

En el artículo 23 del Capítulo IV, perteneciente a las Prioridades, aclara enfáticamente que "no será aceptado ningún requerimiento de Desarrollo o innovación ni admitida Investigación aplicada si no está asegurada la calidad con que los mismos serán llevados adelante" 
De aquí en más hay varias Ordenanzas como las 789, 873 y 890 ( ARGENTINA MINISTERIO DE CULTURA Y EDUCACIÓN, UNIVERSIDAD TECNOLÓGICA NACIONAL, 1995, 1998, 1999) y las Resoluciones 552, 558 y 01 (MINISTERIO DE CULTURA Y EDUCACIÓN, UNIVERSIDAD TECNOLÓGICA NACIONAL, 1999, 2002) en donde, por ejemplo, se norma la carrera del Investigador, los Centros de Investigación y Desarrollo y la propia Secretaria de Ciencia y Tecnología.

Como se puede apreciar, a medida que se avanza en la historia, cada vez son más rápidos los cambios y mayores las urgencias en dedicar el tiempo y el espacio suficiente a la Investigación.

En el año 2002, la UTN manifestó, mediante la Resolución 01/02 (Ministerio de Cultura y Educación, Universidad Tecnológica Nacional, 2002) que había decidido presentarse en forma voluntaria a la acreditación de sus carreras.

Como resultado de esa evaluación, la exigencia de aumentar la actividad de investigación en casi todas las carreras, y hacerlo bajo parámetros de calidad, fue una constante.

Tanto en la Resolución 275 como en los comentarios realizados por la Comisión Nacional de Evaluación y Acreditación Universitaria (CONEAU) se puede ver la importancia que se da a la vinculación internacional. Éstos son motivos más que importantes para buscar una normativa de orden internacional que sirva como guía a la hora de la confección de los proyectos.

Es evidente que existe una problemática asociada a los proyectos de investigación. Para definir mejor el alcance de esta problemática se decidió realizar un relevamiento con los investigadores de las Facultades Regionales Avellaneda y Buenos Aires.

Relevamiento realizado con los investigadores en las Facultades Regionales de Avellaneda y Buenos Aires de la UTN de la metodología para el armado de proyectos

En conversaciones mantenidas con distintos investigadores de ambas Regionales se observó que, de un modo general, éstos no estaban conformes con los formularios actuales para la presentación de los proyectos. Por tal motivo se decidió hacer una encuesta destinada a definir el estado de situación.

Para que la encuesta pudiera ser representativa, fue necesario conocer el total de los investigadores activos y luego aplicar alguna normativa que indicase cuál era la cantidad mínima de encuestados elegidos al azar, que serían representativos de ese total. 
A mediados del 2005 (momento en el que se realizó la encuesta) la cantidad total de investigadores de ambas regionales era de 210. Sobre ese universo se aplicó la Norma IRAM 15 para el tamaño mínimo de encuestas a realizar. En este caso se tomó la condición más desfavorable del nivel S-3 (muestreo especial) que es con un Nivel Aceptable de Calidad (AQL) de 1. Con tales condiciones, la cantidad mínima de encuestas mínimas debería ser al menos 13, lo cual representaba el $6,2 \%$ del total. Con base en este concepto se realizaron en total 15 encuestas, cifra que representa el 7,1\% del total de investigadores y, por la aplicación de la norma, representativo del lote total.

La encuesta fue entregada personalmente a cada investigador en la Facultad Regional Avellaneda, mientras que en la Regional Buenos Aires el Secretario de Ciencia y Tecnología fue quien se encargó de distribuirlas vía correo electrónico.

De las preguntas realizadas surgieron, en relación con cada uno de los ítems detallados, los siguientes comentarios generales:

1 Documentación utilizada para el armado de un nuevo proyecto.

Por lo general los investigadores utilizan el formulario designado para tal fin, pero varios de ellos presentan los proyectos de acuerdo a su propio pensamiento y criterio.

2 Objetivo y características del proyecto.

En este punto, la gran mayoría de los investigadores conoce con claridad qué debe hacer y cómo expresarlo.

\section{El cliente.}

Hay investigadores que presentan proyectos de investigación y no tienen en claro quién será el beneficiario del resultado obtenido.

\section{$4 \quad$ Planificación en etapas.}

Los investigadores en general planifican en forma intuitiva y no metodológica. Es decir, al no existir en el formulario un requisito en tal sentido, algunos no lo tienen en cuenta y otros no saben cómo volcarlo por escrito.

\section{Definición de fases y procesos del proyecto.}

Muy pocos investigadores tienen en cuenta las fases y los procesos del proyecto; se da la misma situación que en la pregunta anterior. 


\section{Sistema de Gestión de la Calidad.}

Los investigadores no tienen en cuenta los sistemas de gestión de calidad, a menos que se lo exijan.

7 Responsabilidades de cada uno de los integrantes del grupo.

Sólo los investigadores que son directores de proyectos tienen en claro las responsabilidades de cada uno de los integrantes del grupo. Se han observado casos de investigadores que, al no ser directores, no sabían qué funciones cumplían otros miembros del grupo.

\section{Gestión de los recursos materiales y humanos.}

El grupo de trabajo solicita los materiales y espera recibirlos para investigar. Su actitud es muy pasiva en ese sentido; prueba de ello es lo que expresó uno de los investigadores en la encuesta: "si nos dan los recursos solicitados investigamos, de lo contrario escribimos".

\section{Estimación de la duración con hitos verificables.}

Esta pregunta esta íntimamente ligada con las preguntas 4 y 5. Las respuestas tienen que ver con el tipo de investigación que está realizando el grupo. Si se esta trabajando, por ejemplo, en un proceso químico, siempre hay mediciones que marcan etapas para seguir avanzando, pero hay otros trabajos, en los cuales los hitos no son tan fácil de ubicar.

\section{Estimación de costos y su metodología de control.}

Por lo general, los investigadores realizan una estimación de costos a la hora de presentar el proyecto, pero luego no tienen una metodología para controlarlos. Podemos agregar que uno de los investigadores expresó que lo importante era que el proyecto fuese aprobado, que una vez lograda esa instancia de una u otra manera siempre al final se conseguirían los recursos, hubieran sido previstos o no en el proyecto original.

\section{Estructura organizacional.}

Los investigadores arman los grupos según sus necesidades, pero por lo general son muy independientes a la hora de trabajar. Los grupos son, en algunos casos, interdisciplinarios y, como manifestaron en una encuesta: "cada uno se ocupa de cumplir con lo que el Director le solicita". Esto se hace sin mantener reuniones entre todos los miembros, lo que provoca que, incluso, haya quienes no conozcan a sus compañeros de proyecto. 


\section{Metodología para las comunicaciones.}

Las comunicaciones existen, pero sólo entre el Director y cada uno de los integrantes de su grupo de investigación. Por lo general no hay reuniones entre todos los miembros del proyecto ni con los de otros grupos.

\section{Informes de avance.}

Por lo general los informes de avance se realizan sólo cuando los solicita la Secretaría de Ciencia y Tecología.

\section{Riesgos del proyecto.}

Los investigadores, en su mayoría, entienden como riesgo únicamente la parte económica, es decir, el riesgo de no recibir los materiales necesarios para poder continuar con el proyecto, y no tienen en cuenta las dificultades que pueden tener a la hora de lograr por ejemplo el objetivo de disminuir un porcentaje determinado, el residuo de un proceso.

\section{Compras.}

Las compras no son gestionadas por los investigadores; ellos sólo solicitan lo que necesitan y eventualmente en algunos casos proponen proveedores que pueden suministrar dichos insumos.

\section{Control de la documentación producida.}

Los investigadores no disponen de una metodología de control de la documentación. Elaboran papeles internos y solamente algunos directores llevan un correcto registro como resultado de su iniciativa individual.

\section{Evaluación de proveedores.}

Los investigadores no realizan la evaluación de los proveedores, ya que no son ellos quienes compran.

\section{Gestión del conocimiento.}

En casi la totalidad de los grupos de investigación hay becarios. Los directores consideran como gestión del conocimiento a la transferencia que reciben los becarios por la participación en los proyectos.

\section{Mediciones, Análisis y Mejoras.}

Las mediciones, los análisis y las mejoras que se reflejan tienen directa relación con el tipo de investigación que se esté llevando a cabo. Hay grupos 
de investigación que deben hacerlo por necesidad y hay quienes lo hacen sólo si les es exigido.

\section{Indicadores.}

En el caso de los indicadores se da la misma situación que la explicada en la pregunta anterior.

Dado que las preguntas fueron abiertas para conocer la opinión de cada investigador y dar así la oportunidad de explayarse en sus opiniones, se hacía difícil poder representar en un gráfico lo expresado en las encuestas.

Por ello se decidió graficar las respuestas teniendo en cuenta como afirmativas o casos favorables (asignando un 1) las que manifestaron al menos hacer algo sobre el tema consultado y como negativas o casos no favorables (asignando un 0) aquéllas en las que no se registraba actividad alguna. Los resultados se indican en la Tabla 1 y la Figura 1.

Tabla 1. Cantidad de casos Favorables y no favorables por pregunta.

\begin{tabular}{|c|c|c|}
\hline Pregunta & Casos favorables & Casos no favorables \\
\hline 1 & 10 & 10 \\
\hline 2 & 13 & 7 \\
\hline 3 & 11 & 9 \\
\hline 4 & 9 & 11 \\
\hline 5 & 7 & 13 \\
\hline 6 & 3 & 17 \\
\hline 7 & 2 & 18 \\
\hline 8 & 6 & 14 \\
\hline 9 & 7 & 13 \\
\hline 10 & 6 & 14 \\
\hline 11 & 6 & 14 \\
\hline 12 & 9 & 11 \\
\hline 13 & 10 & 10 \\
\hline 14 & 6 & 14 \\
\hline 15 & 6 & 14 \\
\hline 16 & 6 & 14 \\
\hline 17 & 5 & 15 \\
\hline 18 & 5 & 15 \\
\hline 19 & 7 & 13 \\
\hline 20 & 5 & 15 \\
\hline
\end{tabular}




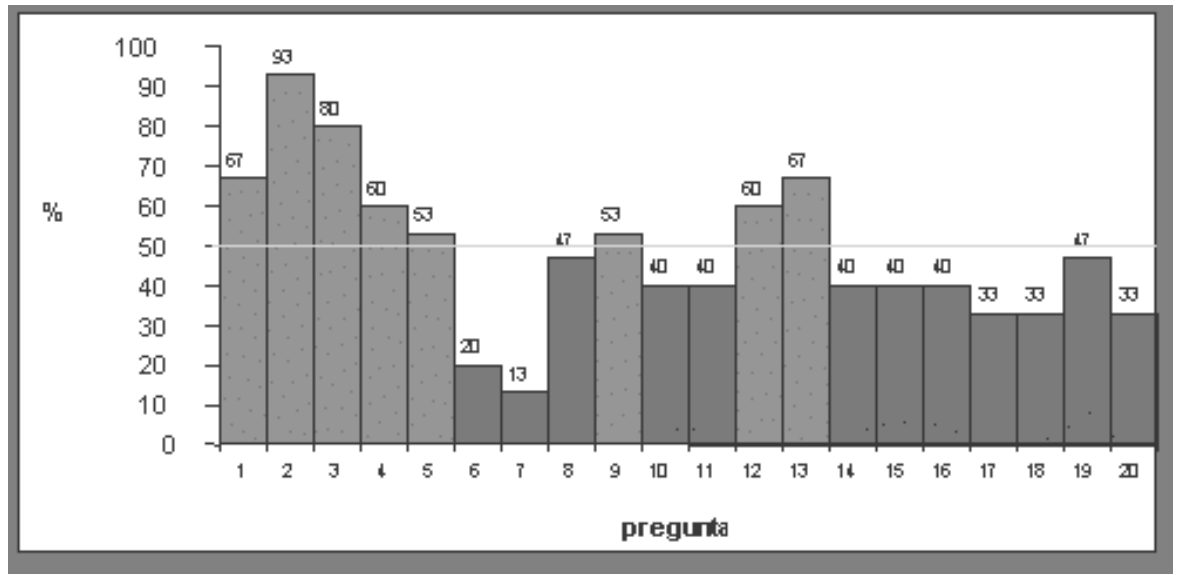

Figura 1: Porcentaje de ocurrencia de casos favorables por pregunta.

Se establece una cota de mínima del 50\%, es decir que aquellas respuestas que se encuentran por encima de este valor cumplen con los requisitos de mínima.

Analizando la Figura 1 y los resultados de la encuesta, se observa que, evidentemente, la gran mayoría de los investigadores no tiene en cuenta la organización de acuerdo a un Sistema de Gestión de Calidad y la definición de las responsabilidades (Preguntas 6 y 7).

Por otro lado, las respuestas a las preguntas $10,11,14,15,16,17,18$ y 20 , si bien muestran mejores indicios que las de las preguntas 6 y 7 , no satisfacen en sus explicaciones lo que debería ser la disciplina de un Modelo de Gestión de la Calidad para proyectos.

Uno de los elementos indispensables detectados en la encuesta es que, por lo general, los directores, al ser en su gran mayoría docentes con varios años de experiencia y con muchos trabajos presentados, conocen exactamente cómo se debe presentar un proyecto según lo requerido por UTN, pero el resto de los investigadores no tiene esta información y desconoce, inclusive, lo que el mismo director ha presentado.

Se ve con esto, por un lado, una falta importante de comunicación entre los miembros de los grupos, responsabilidad ésta del Director del Proyecto y, por el otro, un desconocimiento de los Sistemas de Gestión de la Calidad; por lo general, los investigadores sólo los asocian con la Norma ISO 9001:2000 y su certificación. 


\section{Análisis de la Norma ISO 10006:2003}

A partir de los análisis efectuados aparece una problemática basada en la necesidad de una sistematización de los requisitos y registros para el armado de un proyecto.

Tal como se ha mencionado, de la búsqueda de una normativa que sea aplicable y adecuada para esta problemática surge que la Norma ISO 10006:2003 es la que mejor se adapta a la necesidad planteada.

Se analizó cada capítulo de la Norma, se explicó el estado actual de cumplimiento y la forma en que debería interpretarse en el ámbito de la UTN.

Hay, por ejemplo, una diferencia muy marcada entre la visión de lo que es un proyecto para esta Universidad y la definición que da la norma citada. En el primer caso, la Resolución 232/98 de UTN expresa que proyecto es:

Conjunto coordinado de tareas científico tecnológicas que comprenden total o parcialmente actividades de investigación y desarrollo y que, comenzando por conocimientos preexistentes, permite llegar a un objetivo cuyas características han sido previamente determinadas. Por otra parte, para la ISO 10006:2003 proyecto es:

"Proceso único consistente en un conjunto de actividades coordinadas y controladas con fechas de inicio y de finalización, llevadas a cabo para lograr un objetivo conforme con requisitos específicos, incluyendo las limitaciones de tiempo, costo y recursos.

Del análisis de las dos definiciones se desprende que, si bien para UTN los proyectos están acotados en tareas científicas y tecnológicas, no se menciona en ningún momento la duración, sus costos y los recursos. Estos elementos son fundamentales para cualquier tipo de seguimiento y control, mas allá de lo útil que puede resultar al investigador en el ordenamiento de su trabajo.

\section{Resultados}

\section{El Modelo y su validación}

Para el armado de un modelo que sirviera como guía había dos caminos posibles:

1) Desarrollarlo con base en las directrices de la Norma en cuestión, ignorando lo existente. 
2) Tomando como base los formularios vigentes, realizar las adecuaciones necesarias para el cumplimiento de los requisitos de la ISO 10006:2003.

Se consideró que era mejor elegir el segundo camino ya que, seguramente, esos formularios eran el producto de muchas horas de trabajo y dedicación de personas a quienes no gustaría que todo lo realizado fuese ignorado. Esto es importante, porque son estas mismas personas las que en el futuro podrían utilizar el resultado de este trabajo.

Otro punto relevante es que, con los formularios actuales, se presentaron muchos proyectos que llegaron a conclusiones satisfactorias, por cuya razón se entiende que no deben ser descartados, sino rescatados y mejorados. Existen también algunos aspectos que son significativos para la UTN y que necesita conocer aun cuando no son considerados en la Norma, como, por ejemplo, los datos relacionados con la categorización del investigador.

Adicionalmente, y teniendo en cuenta que el presente trabajo estaba destinado a la elaboración de una propuesta para mejorar la calidad de la presentación de los proyectos dentro de la UTN, resultaba importante partir de la base ya existente y proponer desde allí modificaciones y agregados. De esta forma se concebía que el resultado debía ser un modelo menos traumático en su utilización y más amigable para los investigadores ya familiarizados con los formularios actuales.

Para validar los formularios desarrollados se decidió aplicarlos a dos proyectos de la Facultad Regional Avellaneda y a dos de la Facultad Regional Buenos Aires. A los efectos de obtener información mas representativa de esta experiencia, se eligió en cada Regional un proyecto que estuviera operativo, conducido por Director investigador con mucha experiencia y otro que recién estuviera por comenzar, con un Director no tan familiarizado con los formularios existentes.

Debido a que la información contenida en los proyectos es confidencial, no es posible dar información acerca de los proyectos sobre los que han sido validados los formularios, de forma tal que para preservar el anonimato de los investigadores, los comentarios serán identificados como pertenecientes a Grupos (1, 2, 3 y 4) sin especificar a qué Regional corresponden, ni tampoco a qué proyecto.

Los comentarios de los investigadores fueron: 
Grupo 1

El nuevo formulario es semejante al vigente en algunos aspectos, pero a diferencia de los anteriores, éstos permiten y exigen una diferenciación por etapas de la realización del proyecto. Esto nos exige un mayor esfuerzo a la hora de la presentación pero lo consideramos oportuno, al igual que el análisis de riesgo.

Grupo 2

No hemos tenido mayores inconvenientes en el llenado del formulario. Sí, se nos hace sumamente dificil conseguir los avales, por ser nuestro proyecto algo innovador en la Regional y no tener expertos en el tema. No nos ha quedado claro si el Director y el Co-Director deben completar los datos del personal involucrado, ya que estas planillas deben ser firmadas por ellos mismos.

Grupo 3

En nuestro proyecto, todos los integrantes consideramos que todo lo que tenga que ver con la Calidad debe ser bienvenido. Hemos sido "víctimas" de todas las modificaciones que han tenido los formularios vigentes y entendemos que todas fueron realizadas con la mejor de las intenciones pero nunca nos fue consultado qué opinamos sobre los mismos.

Habiendo utilizado estos nuevos formularios quisiéramos aprovechar para dar nuestro punto de vista respecto de la Idea Proyecto. No estamos en desacuerdo con la información que en ellos se solicita, si estamos en desacuerdo que se pida lo mismo en la presentación del PID ${ }^{2}$. De hecho muchas veces nos piden que entreguemos junto la IP y el PID. En tal sentido propondríamos la eliminación de los formularios de IP para no duplicar en forma innecesaria la información que nos requieren.

Por otro lado nos pareció muy bueno y útil la identificación de los riesgos. En los proyectos que venimos realizando, si bien nunca nadie nos lo exigió, aplicamos una metodología de identificación de riesgos pero sin un fundamento sólido como en este caso.

\section{Grupo 4}

Debido a que es nuestra primera experiencia en la presentación de proyectos nos surgieron varias dudas, a saber:

$2 \quad$ Proyecto de Investigación y Desarrollo. 
- ¿El formulario de IP y PID debe ir firmado por el Área Académica antes de ser presentado a la Secretaría de Ciencia y Técnica?

- ¿Si no conseguimos 2 avales cientificos no podemos presentar el proyecto?

- ¿Las etapas del proyecto pueden ser más de 6?

- ¿Puede un proyecto demorar más de 3 años?

- ¿El Director del Departamento de la Especialidad no debe dar su opinión sobre el proyecto?"

\section{El Modelo}

Habiendo validado el Modelo, se procedió a realizar las modificaciones sugeridas y aquéllas que se consideraron oportunas a los efectos de mejorar y optimizar los formularios y la guía.

Por otra parte, más allá de los formularios, el procedimiento y la guía, que son para el investigador, la Secretaría de Ciencia y Tecnología debería llevar los siguientes indicadores en función de los proyectos presentados:

- Proyectos aprobados en relación con proyectos presentados: Este indicador determinará, por ejemplo, el grado de especialización de los investigadores a la hora de presentar un proyecto de investigación y desarrollo.

- Proyectos abandonados en relación con proyectos aprobados: Aquí se puede determinar si es necesario tomar algún tipo de acción sobre los motivos por los cuales los proyectos no llegan a cumplir su objetivo.

- Valores presupuestarios promedio de los proyectos presentados: Con este dato se puede realizar el flujo de caja necesario para I+D en cada Facultad Regional.

- Cantidad de proyectos por Departamentos y por Áreas: Esto permite detectar los sectores de mayor investigación y también aquéllos a los que se debe ayudar para mejorar su rendimiento.

- Cantidad de proyectos de investigación que cumplen con las líneas propuestas por el Ministerio de Educación, Ciencia y Tecnología: Con este indicador se puede observar cuán alejada está la investigación que se realiza en la Universidad Tecnológica de la necesidad planeada desde el Gobierno Nacional.

El Modelo, como ya fue mencionado, consta de un procedimiento, una guía y doce formularios. Dada la extensión del Modelo completo, se detallará a continuación sólo el procedimiento. 


\section{Procedimiento para el armado de los proyectos de Investigación y Desarrollo}

\section{Objetivo y alcance}

Establecer un procedimiento para la planificación, la realización y el armado de los proyectos a ser presentados en el ámbito de la Universidad Tecnológica Nacional. El mismo es de aplicación a todas las Facultades Regionales y Unidades Académicas que posean grupos de investigación.

\section{Procedimiento}

Este procedimiento consta de 5 procesos, cuyas generalidades son:

- Planificación de los recursos: Los procesos relativos a los recursos tienen como finalidad planificar y controlar los mismos. Algunos ejemplos de recursos son: equipos, instalaciones, materiales, software, personal, servicios y espacio.

- Establecimiento de la estructura organizativa del proyecto: Debido a que tanto la calidad como el éxito de un proyecto dependen del personal que participe en el mismo es fundamental que el grupo de trabajo quede claramente constituido y sus responsabilidades claramente delineadas.

- Definición de las Actividades: El alcance del proyecto incluye la descripción del producto, de las distintas etapas del proyecto, sus características y el modo en que han medirse o evaluarse.

- Identificación y evaluación del Riesgo: Para la Norma ISO 10006:2003 el término "riesgo" se utiliza en el mismo sentido que "incertidumbre", es decir, considerando aspectos tanto negativos como positivos. En tal sentido se debe identificar los riesgos, evaluarlos, proponer el tratamiento y controlarlos.

- Proceso relacionado con la mejora, la medición y el análisis: Este proceso proporciona orientación sobre el modo en que el grupo de investigación debería aprender de su propio proyecto.

\section{Proceso 1:}

Planificación de los recursos.

Se deben identificar los recursos necesarios para el proyecto. Esto es:

- Qué recursos 
- $\quad$ Cuándo se requerirán

- Cómo, es decir de que modo

- $\quad$ De dónde se obtendrán los recursos

Deberán explicitarse, mediante los formularios correspondientes, los recursos, incluyendo la estimación, los montos y las limitaciones.

Documentación de Referencia

Norma ISO 10006:2003 sección 6.1.2

Formularios Asociados

F-06- A Procesos Relacionados con el Costo (Equipamiento)

F-07- A Procesos Relacionados con el Costo (Servicios no personales)

F-08- A Procesos Relacionados con el Costo (Bienes de Consumo)

F-09- A Procesos de Compras

\section{Proceso 2:}

Establecimiento de la estructura organizativa del proyecto.

La estructura organizativa del proyecto deberá diseñarse con el objeto de fomentar una comunicación y una cooperación eficaces y eficientes entre todos los participantes en el proyecto.

Deberán elaborarse y documentarse descripciones de los puestos o funciones, incluyendo las atribuciones de responsabilidad y autoridad.

Documentación de Referencia

Norma ISO 10006:2003 sección 6.2.2

Formularios Asociados

F-04- A Nomina del Personal involucrado

F-05- A Datos del Personal involucrado

\section{Proceso 3:}

\section{Definición de las actividades}

El proyecto deberá estructurarse sistemáticamente en actividades realizables para cumplir con el objetivo de la investigación/desarrollo.

Cada actividad deberá definirse de forma tal que sus resultados sean medibles. 
Documentación de Referencia

Norma ISO 10006:2003 sección 7.3.4

Formularios Asociados

F-02- A Planeamiento del Proyecto

F-03- A Plan de Actividades por Fase

\section{Proceso 4:}

Identificación y evaluación de los riesgos

La identificación de los riesgos se deberá realizar al comienzo del proyecto, durante las evaluaciones del avance y en otras ocasiones en las que se tomen decisiones importantes.

La evaluación de los riesgos es el proceso de analizar y valorar los riesgos identificados para las distintas etapas del proyecto.

Deberán evaluarse todos los riesgos identificados. En esta evaluación deberán tenerse en cuenta la experiencia y los datos históricos de proyectos anteriores.

Deberán determinarse los criterios y las técnicas que habrán de utilizarse en la evaluación.

Deberá realizarse un análisis cualitativo, al cual debería seguir un análisis cuantitativo siempre que sea posible.

Documentación de Referencia

Norma ISO 10006:2003 secciones 7.7.2 y 7.7.3

Formularios Asociados

F-10- A Procesos Relacionados con el Riesgo (Guía)

F-11- A Procesos Relacionados con el Riesgo

\section{Proceso 5:}

Proceso relacionado con la Mejora, la Medición y el Análisis

Se deberán utilizar los resultados de la medición y el análisis de los datos derivados de las fases del proyecto y aplicar acciones correctivas, acciones preventivas y métodos para la prevención de pérdidas.

La Secretaría de Ciencia y Tecnología necesita asegurarse de que la medición, la recopilación y la validación de datos son eficaces y eficientes para mejorar el 
desempeño del grupo de investigación e incrementar la satisfacción del cliente $\mathrm{y}$ otras partes interesadas.

Documentación de Referencia

Norma ISO 10006:2003 secciones 8.1 y 8.2

Formularios Asociados

F-12- A Procesos Relacionados con las mediciones.

\section{Registros}

El Director del proyecto es el máximo responsable del mantenimiento de los registros del proyecto, incluyendo carpetas, planes, programas e informes de revisión por un tiempo mínimo de 5 años a partir de la finalización del proyecto y que se ha entregado toda la documentación a la Secretaría de Ciencia y Tecnología de la UTN.

\section{Conclusiones}

\section{Análisis del los resultados de la aplicación del Modelo}

Del análisis de las encuestas realizadas a los investigadores y de los comentarios recibidos en distintas reuniones mantenidas con autoridades de las distintas Facultades Regionales de la UTN y con los propios investigadores surgieron las siguientes posibilidades de mejora sobre los formularios:

- Dar de baja al formulario de Idea Proyecto.

En principio, es correcto lo expresado por los investigadores, respecto a que de alguna manera se les está solicitando dos veces la misma información. Esto se percibió ni bien se comenzó el trabajo; sucede que, como ya fue explicado, la idea no era dar de baja nada de lo existente sino hacer todos los esfuerzos para adecuarlo a lo que solicita la Norma ISO 10006:2003.

Por otro lado, cuando un proyecto es analizado y evaluado, siempre es aconsejable que quienes realizan esta labor dispongan de toda la información del mismo. De lo contrario, bajo el régimen actual, podría darse el caso que una IP sea aprobada pero, luego de su evaluación, el PID sea rechazado. Tal situación genera las siguientes dificultades:

- Dos procesos de evaluación. 
- Mucho tiempo de demora en tomar la decisión definitiva de aceptar o no al PID.

- Doble costo en informar el resultado de ambas evaluaciones, en convocar a los evaluadores, en el armado de las resoluciones y en la administración de la documentación.

- Desgaste de los investigadores.

Si bien la IP y el PID son dos instancias distintas; la primera funciona como un filtro previo que, bajo esta figura, podría ser realizada en informalmente por el Secretario de Ciencia y Tecnología cuando el grupo investigador tiene el primer bosquejo del proyecto.

- Modificar el requisito del Aval Científico.

Existe un problema en relación con el requisito solicitado en los formularios y es que no son muchos los investigadores categorizados que hay en cada una de las Facultades Regionales y Unidades Académicas. En tal sentido se da el caso de que, concretamente, en algunas FR el investigador que más conocimiento tiene sobre una temática en particular es el mismo que está presentando el proyecto y el único con el que cuenta. Esto hace que sea imposible conseguir dos avales científicos de individuos que puedan opinar sobre el trabajo presentado con un conocimiento específico y calificado en el ámbito de esa casa de estudios.

La propuesta para solucionar esta problemática, al menos en el ámbito de las Facultades Regionales del Área Metropolitana (Incluye a las Regionales Avellaneda, Buenos Aires, Delta, Haedo, General Pacheco y La Plata), es elaborar un banco de datos con los investigadores categorizados de toda el Área Metropolitana y que del mismo el investigador pueda elegir a un par suyo de otra Facultad Regional para que le de el aval necesario.

Esta metodología puede ser aplicada perfectamente en otras zonas del país donde la diferencia entre cantidad de investigadores de las distintas Regionales y Unidades Académicas es mucho más marcada.

Debido a la problemática planteada se considera oportuno permitir la presentación del PID con un solo aval.

- Modificar el Formulario de Índice de Fases.

La idea es que sea posible presentar "n" fases, pero nunca menos de tres.

- Modificar la explicación del llenado de los datos personales.

El propósito de este requisito es asegurar que tanto el Director como el CoDirector deban completar el mismo formulario que el resto del personal. 
Todas estas posibilidades de mejora fueron aplicadas al Modelo definitivo.

El resultado de este trabajo podrá ser aplicado por los investigadores y las áreas de Investigación y Desarrollo donde se desarrollarán esos proyectos para el armado, control y seguimiento de los mismos. Esto les permitirá a los investigadores dedicarse netamente a su tema de investigación sabiendo que no omitirán elementos indispensables de gestión y control y que desde la presentación trabajarán bajo una normativa de Gestión de la Calidad.

\section{Propuestas a futuro}

Se ha detectado, a partir de distintas reuniones que se han mantenido con los Secretarios de Ciencia y Tecnología de la UTN, que existe un problema dentro de las distintas Facultades Regionales, en relación con el proceso de evaluación de los proyectos.

Las autoridades se ven ante la necesidad de aumentar la cantidad de proyectos de $\mathrm{I}+\mathrm{D}$, requisito impuesto en forma casi general por la CONEAU a la hora de la acreditación, y esto hace que de alguna forma determinados proyectos sean "impuestos". Esto quiere decir que, en realidad, como no existe un proceso de evaluación independiente y externo a la FR donde se presentan, los proyectos no son evaluados exhaustivamente, ya que de alguna manera son las mismas autoridades las que quieren su aprobación sí o sí, para aumentar de esta manera la cantidad y cumplir con lo exigido por la CONEAU.

Es evidente que resulta necesario que la UTN aumente la "cantidad" de los proyectos, pero debe dedicarle especial atención a la "calidad" de los mismos y esto se logra comenzando con un buen proceso de evaluación, que debería iniciarse en cada FR o UA como paso previo a la presentación en la SCyT del Rectorado de la UTN.

Para solucionar este inconveniente, al menos en el Área Metropolitana, se propone la creación de un Consejo Asesor integrado por dos miembros (Investigadores categoría 1, 2 o 3 del Ministerio de Educación, Ciencia y Tecnología o categoría A, B o C de la UTN) de cada una de las Facultades Regionales de la mencionada área. Dicho Consejo tendría la misión de evaluar todos los proyectos que se presenten en las regionales pertenecientes al área y su dictamen se elevaría al Consejo Académico de la Facultad Regional de origen (Esta metodología ha sido aceptada por el AM luego de la propuesta presentada por el autor en la reunión de Secretarios de Ciencia y Tecnología realizada en la Facultad Regional Delta el 23-03-06). 
Otro problema detectado durante la elaboración de la tesis es que las actitudes transcurren como si lo realmente importante no fuera el "resultado" del proyecto en sí, sino el hecho de tenerlo aprobado y aumentar la cantidad de proyectos en ejecución. Prueba de esto es que no existe un correcto seguimiento de los proyectos en el Rectorado de la UTN y ni mucho menos en las Facultades Regionales.

Se entiende que el resultado de esta tesis debería tomarse como base para continuar con un trabajo similar dedicado al seguimiento y al análisis postproyecto. Esto último no sólo no se realiza, sino que directamente no existe en el ámbito de la UTN. Se considera que sería de suma utilidad contar con este tipo de análisis, porque permitiría retroalimentar al sistema en función de los resultados obtenidos.

La evaluación post-proyecto es fundamental para la gestión, ya que permite evaluar entre otras cosas cómo funcionó la organización, si se cumplió con los requisitos, si las etapas fueron correctamente pautadas, si cada uno de los responsables cumplió su tarea tal cual lo estipulado, etc.

Esta información permitiría trabajar en la Mejora Continua desde el comienzo, con el mismo formulario de presentación de PID, haciendo las modificaciones que los resultados demuestren necesarias para su presentación y seguimiento.

Algo que atenta contra el éxito de cualquier SGC que quiera ser implementado es la situación que se presenta debido a la falta de investigadores categorizados que puedan dirigir los proyectos. Nótese que, para ser aceptado en esta función, el investigador debe tener categoría 1, 2 o 3 del Ministerio de Educación, Ciencia y Tecnología o A, B o C de la UTN. Como en las Facultades Regionales son pocos los investigadores que cumplen con estos requisitos, sucede que los que sí los cumplen terminan siendo directores de varios proyectos. Tal situación hace que una de las funciones principales del director, que es ser el "líder" del proyecto, no se cumpla y solo figure y "preste" su nombre y categoría para que el mismo sea aprobado y de esta manera no sienta ningún otro compromiso con el grupo de investigación.

El hecho de que los directores de proyecto no sean los líderes es muy grave, porque de ellos depende el control, la realización de los informes de los estados de avance, las posibilidades de mejoras, las necesidades, etc.

Esta situación fue relevada en la reunión de Secretarios de Ciencia y Tecnología de la UTN que se realizó en la FR Santa Fe el 5 y 6 de Abril del 2006 y no tiene otra salida que no sea aumentar la cantidad de investigadores calificados y calificar a los existentes. 
Otra posibilidad de mejora del sistema es la creación de una base de datos donde figuren todos los proyectos de las distintas Facultades Regionales y Unidades Académicas de la UTN, junto con su estado de avance. El día que esta información exista y esté disponible para cualquier investigador, será una herramienta fundamental para mejorar la investigación, ya que les permitirá tomar la experiencia de otros. Sería también in factor coadyuvante en el establecimiento de contactos, por iniciativa propia, entre investigadores. Se ha visto, en la mencionada reunión, que varias FR están investigando sobre los mismos temas, lo que demuestra una muy mala comunicación entre los grupos de investigación de la UTN.

De la encuesta realizada a los investigadores surgen otras posibilidades de mejora del sistema. Concretamente, como respuesta a las preguntas 8,10 y 11 , se puede notar que hay una actitud por demás pasiva, esto es que no se ve que los investigadores tomen la actividad de investigación como una tarea principal sino como algo secundario que hacen cuando pueden y con lo que puedan.

Esto se debe a que, por un lado, el presupuesto que se destina dentro de la Universidad Tecnológica Nacional a Ciencia y Tecnología es exiguo y, por el otro lado, no hay una política clara del propósito y el destino de la investigación en esta Universidad para acompañar al crecimiento del país.

En tal sentido se considera que la Secretaría de Ciencia y Tecnología y la Secretaría Académica del Rectorado deberían trabajar en conjunto para establecer una política de investigación que tenga que ver con las incumbencias de las carreras que ahí se dictan, con el postgrado y con las necesidades del país.

\section{Agradecimientos}

El autor desea agradecer a la Ing. Maria Marta Mazzini y al Mg. Ricardo Brunetti, por su apoyo científico, esencial para llevar a buen término la tesis sobre la que se realizó este trabajo, pero también por la ayuda brindada para exponer de manera clara las ideas que se quería expresar. 


\section{Referencias}

ARGENTINA. Ministerio de Cultura y Educación, Universidad Tecnológica Nacional. Rectorado. Estatuto de la Universidad Tecnológica Nacional. Artículo 12. Bueno Aires, 1998. p. 3.

ARGENTINA. Ministerio de Cultura y Educación, Universidad Tecnológica Nacional. Rectorado. Ordenanza 665, primer Capítulo del Anexo II, Artículo 1. Bueno Aires, 1989.

ARGENTINA. Ministerio de Cultura y Educación, Universidad Tecnológica Nacional. Rectorado. Ordenanza 703, Artículo 1. Bueno Aires, 1991.

ARGENTINA. Ministerio de Cultura y Educación, Universidad Tecnológica Nacional. Rectorado. Resolución 275, Anexo 1. Bueno Aires, 1995.

ARGENTINA. Ministerio de Cultura y Educación, Universidad Tecnológica Nacional. Rectorado. Resolución 232, Anexo 1. Bueno Aires, 1998.

ARGENTINA. Ministerio de Cultura y Educación, Universidad Tecnológica Nacional. Rectorado. Resolución 232, Anexo 1, Artículo 21. Bueno Aires, 1998 p. 17.

ARGENTINA. Ministerio de Cultura y Educación, Universidad Tecnológica Nacional. Rectorado. Resoluciones 552 y 558. Bueno Aires, 1999.

ARGENTINA. Ministerio de Cultura y Educación, Universidad Tecnológica Nacional. Rectorado. Ordenanzas 789, 873 y 890. Bueno Aires, 1995, 1998, 1999.

ARGENTINA. Ministerio de Cultura y Educación, Universidad Tecnológica Nacional. Rectorado. Resolución 01, Artículo 1. Bueno Aires, 2002.

ISO 9000:2000. Sistemas de Gestión de Calidad. Fundamentos y vocabulario.

ISO 9001:2000. Sistemas de Gestión de Calidad. Requisitos.

ISO 9004:2000. Sistemas de Gestión de Calidad. Directrices para la mejora de desempeño.

ISO 10006:2003. Sistemas de Gestión de Calidad. Directrices para la gestión de calidad en los proyectos. 